\title{
Communicating vessels: a non-linear dynamical system
}

\author{
Vasos comunicantes: um sistema dinâmico não linear \\ Roberto De Luca*1, Orazio Faella ${ }^{1}$ \\ ${ }^{1}$ Dipartimento di Fisica "E. R. Caianiello", Università degli Studi di Salerno, Fisciano SA, Italy
}

Received on December 22, 2016. Revised on January 26, 2017. Accepted on February 05, 2017.

The dynamics of an ideal fluid contained in two communicating vessels is studied. Despite the fact that the static properties of this system have been known since antiquity, the knowledge of the dynamical properties of an ideal fluid flowing in two communicating vessels is not similarly widespread. By means of Bernoulli's equation for non-stationary fluid flow, we study the oscillatory motion of the fluid when dissipation can be neglected.

Palavras-chave: Bernoulli's equation, non-stationary fluid, fluid dynamics

Estuda-se a dinâmica de um fluido ideal contido dentro de dois vasos comunicantes. Embora as propriedades estáticas desse sistema sejam conhecidas desde a Antiguidade, são bem menos conhecidas as propriedades dinâmicas do fluxo de dois fluidos ideais contidos vasos comunicantes. Utilizando a equação de Bernoulli para um fluxo não estacionário, nós estudamos o movimento oscilatório do fluido na ausência de dissipação.

Keywords: equação de Bernoulli, fluxo não estacionário, mecânica dos fluidos.

\section{Introduction}

The topic of communicating vessels is often adopted as a common example in physics teaching [1]. The static property of this system is also used in other subject areas as, for example, sociology and economics [2] and in metaphoric expressions. In fact, it is a widespread common knowledge that a fluid in adjacent containers reaches the same height, measured with respect to a common reference point, independently of the shape of the vessels. However, reference to the dynamical properties of these types of physical systems in physics teaching books is rarely found. One of this rare examples can be found in ref. [3], where it is reported that the height of an ideal fluid contained in a U-shaped tube is seen to oscillate harmonically with a frequency $\omega$ given by:

$$
\omega=\sqrt{\frac{2 g}{L}}
$$

$L$ being the total length of the fluid in the tube. Therefore, although the dynamics of these systems

*Endereço de correspondência: rdeluca@unisa.it have been studied in quantum mechanical and classical contexts [4-6], it is still not much studied at elementary levels. In particular, we would like to mention a specific technological application of this system in structural engineering [6]. In this specific field, U-tube-like containers, called tuned-liquid column dampers, are used to mitigate earth-quake induced vibrations in tall buildings.

In the present work we thus consider the dynamics of two communicating vessels of unequal crosssectional areas, say $S_{1}$ and $S_{2}$, as those shown in Fig.1 . Therefore, the mechanical properties of the system shown in Fig. 1 are analyzed by means of Bernoulli's equation for non-stationary states. We write the nonlinear differential equations governing the motion of the system and point out that the result in equation (1) can be obtained by taking two equal sections; i.e., by writing $S_{1}=S_{2}$. In this case a harmonic solution is obtained. We also notice, by a phase plane numerical analysis, that the solutions for the heights $y_{1}$ and $y_{2}$ is still periodic in the most general case $S_{1} \neq S_{2}$. In this general dynamical system the fixed point is identified with the static 


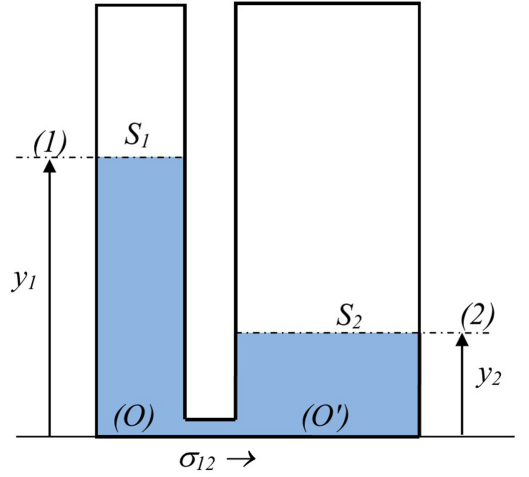

Figura 1: Communicating vessels containing an ideal fluid out of equilibrium. The first vessel has cross-sectional area $S_{1}$ and the fluid column reaches an height $y_{1}$. The second vessel has cross-sectional area $S_{2}$ and the fluid in it is at an height $y_{2}$. The connection channel is positioned at the base of the two vessels and is supposed to be sufficiently small.

equilibrium solution; i.e., the common height $y_{e}$ of the two fluids. Further generalizations are proposed as, for example, the possibility of allowing leakage from the system.

\section{Preliminary dynamical notions}

We start by writing the continuity equation for the fluid [5]:

$$
\begin{aligned}
& S_{1} \dot{y}_{1}+\sigma_{12}=0, \\
& S_{2} \dot{y}_{2}-\sigma_{12}=0,
\end{aligned}
$$

where $\sigma_{12}$ is the flux flow rate in the connecting channel, as shown in Fig. 1. Summing the above equations, we have:

$$
S_{1} \dot{y}_{1}+S_{2} \dot{y}_{2}=0 .
$$

In equation (3) the static solution is implicitly defined. In fact, by integrating with respect to time, we get:

$$
S_{1} y_{1}^{(0)}+S_{2} y_{2}^{(0)}=\lambda\left(S_{1}+S_{2}\right),
$$

where $\lambda$ is the equilibrium height.

In order to tackle the dynamical problem, we need to specify the type of fluid we are dealing with. In what follows we shall thus assume that the fluid is ideal and that Bernoulli's equation, written for the non-stationary regime of the fluid, holds. A derivation of this equation can be found in ref. [7]. Naturally, different behaviour is expected when the fluid is not ideal. We therefore write, for two points in the fluids, one at an height $y_{1}$, corresponding to the free liquid surface in the left container, the second at $y_{0}$, corresponding to point $O$ in Fig. 1:

$$
\int_{y_{0}}^{y_{1}} \frac{\partial v}{\partial t} d y+\frac{1}{2} v_{1}^{2}+\frac{p_{1}}{\rho}+g y_{1}=\frac{1}{2} v_{0}^{2}+\frac{p_{0}}{\rho}+g y_{0},
$$

Setting $p_{1}=p_{a}$ and $v_{1} \approx v_{0}$ in equation (5), we have:

$$
\frac{p_{a}-p_{0}}{\rho}=-y_{1} \ddot{y}_{1}-g y_{1},
$$

where we have assumed that the velocity profile does not appreciably vary with the height $y$ and where the dot stands for "derivative with respect to time". Similarly, for the second vessel, by considering one point corresponding to the free liquid surface and the other at $y_{0^{\prime}}$, we may set:

$$
\frac{p_{a}-p_{0^{\prime}}}{\rho}=-y_{2} \ddot{y}_{2}-g y_{2} \text {. }
$$

In this second case, we have considered $p_{2}=p_{a}$ and $v_{2} \approx v_{0^{\prime}}$. By now combining equations (6) and (7), we obtain:

$$
\frac{p_{0}-p_{0^{\prime}}}{\rho}=y_{1} \ddot{y}_{1}-y_{2} \ddot{y}_{2}+g\left(y_{1}-y_{2}\right) .
$$

Because of equation (3), we have $\ddot{y}_{2}=-\left(S_{1} / S_{2}\right) \ddot{y}_{1}=$ $-\gamma \ddot{y}_{1}$, with the obvious definition of the parameter $\gamma$. On the same token, by equation (4), we may also set

$\gamma y_{1}+y_{2}=k=\lambda(1+\gamma) \Longrightarrow y_{2}=-\gamma y_{1}+\lambda(1+\gamma)$.

Therefore, equation (8) can be written only in terms of the variable $y_{1}$ as follows:

$$
\begin{aligned}
\frac{p_{0}-p_{0^{\prime}}}{\rho} & =(1+\gamma)\left[(1-\gamma) y_{1}+\lambda \gamma\right] \ddot{y}_{1} \\
& +g(1+\gamma)\left[y_{1}-\lambda\right] .
\end{aligned}
$$

If we now go in horizontal from point $O$ to point $O^{\prime}$, assuming the connection channel to be sufficiently small, equation (5) gives:

$$
\frac{1}{2} v_{0}^{2}+\frac{p_{0}}{\rho}=\frac{1}{2} v_{0^{\prime}}^{2}+\frac{p_{0^{\prime}}}{\rho},
$$

so that, because $v_{1} \approx v_{0}$ and $v_{2} \approx v_{0^{\prime}}$, we may rewrite equation (11) as follows:

$$
\frac{p_{0}-p_{0^{\prime}}}{\rho}=\frac{1}{2}\left(\dot{y}_{2}^{2}-\dot{y}_{1}^{2}\right)=\frac{1}{2}\left(\gamma^{2}-1\right) \dot{y}_{1}^{2} .
$$

Finally, by combining equation 100 and equation (12), we have:

$$
\left[(1-\gamma) y_{1}+\lambda \gamma\right] \ddot{y}_{1}+\frac{1-\gamma}{2} \dot{y}_{1}^{2}+g\left[y_{1}-\lambda\right]=0 .
$$


By now making the change variable $y=y_{1}-\lambda$, we may rewrite equation (13) as follows:

$$
\frac{(1-\gamma) y+\lambda}{g} \ddot{y}+\frac{(1-\gamma)}{2 g} \dot{y}^{2}+y=0
$$

For $\gamma=1$ we find the already known result [3]:

$$
\ddot{y}+\frac{g}{\lambda} y=0 .
$$

In this way, for $\gamma=1$, the liquid is seen to oscillate with a period $T=2 \pi \sqrt{\frac{\lambda}{g}}$. A trivial solution $y_{2}=\lambda$ can be found for $\gamma=0$. For $0<\gamma<1$, on the other hand, we can proceed in normalizing the variable $y$ and $t$ as follows:

$$
\begin{gathered}
\xi=\frac{y}{\lambda}, \\
\tau=\sqrt{\frac{g}{\lambda}} t .
\end{gathered}
$$

In this way, we obtain the following non-linear secondorder ordinary differential equation:

$$
[\varepsilon \xi+1] \xi^{\prime \prime}+\frac{1}{2} \varepsilon \xi^{\prime 2}+\xi=0,
$$

where $\varepsilon=1-\gamma=1-S_{1} / S_{2}$, and the prime stands for "derivative with respect to $\tau$ ". The above equation can be cast in the form of two first-order differential equations as follows:

$$
\begin{gathered}
\xi^{\prime}=v, \\
v^{\prime}=-\frac{\xi+\varepsilon v^{2} / 2}{\varepsilon \xi+1} .
\end{gathered}
$$

Equations (18a) and (18b), under the condition $0 \leq \varepsilon<1$, are sufficient to describe the dynamics of the system. Notice that, for $\varepsilon=0$, the harmonic oscillator equation is obtained from 18a and (18b).

\section{Analysis of the dynamical system}

By considering equation (18a) and (18b) we can start by describing the dynamical propertied of the system by means of a phase-plane analysis. In fact, by noticing that equation (15) gives a periodic solution of the problem, we expect that, at least for small values of the quantity $\varepsilon=1-\gamma$, the solution $\xi=\xi(\tau)$ of equation (17) to be periodic with an attraction point corresponding to $(\xi, v)=(0,0)$. In order to detect this property, let us consider the phase-plane representation of the coupled first-order ordinary differential equations (18a and (18b). By considering the initial conditions

$$
\xi(0)=\frac{y_{1}(0)-\lambda}{\lambda}=\xi_{0}, \quad v(0)=0,
$$

we preliminary notice that the value of $\xi_{0}$ must fall within the interval $[-1,1]$. In fact, the dimensionless quantity $\xi$ represents the normalized deviation from the equilibrium position of the height of the liquid in the first column. In this way, we take the fluid to be initially at rest and completely contained in the right vessel or in the left vessel for $\xi_{0}=-1.0$ and $\xi_{0}=+1.0$, respectively. As shown in Fig. 2a and 2b, obtained by means of the software Mathematica, the system shows periodical solutions, since the curves are closed. In Fig. 2a we report solutions for $\xi_{0}=$ +1.0 and for different values of $\gamma$, corresponding to different colours. In Fig. 2b, on the other hand, solutions for $\xi_{0}=0.75$ are shown for the same values of $\gamma$ as in Fig. 2a. The black dashed line represents, in both Figs $2 \mathrm{a}$ and $2 \mathrm{~b}$, the harmonic solution to equation (15). We notice that, for decreasing values of $\xi_{0}$, the $v$ vs. $\xi$ curves tend to collapse on the harmonic solution.

Another useful hint on the periodicity of the solutions of equation (17) can be given by perturbation analysis. In fact, by writing

$$
\xi(\tau) \approx \xi^{(0)}(\tau)+\varepsilon \xi^{(1)}(\tau),
$$

where $\xi_{0}(\tau)$ and $\xi_{1}(\tau)$ are functions to be found, we may rewrite equation (17) as follows:

$$
\begin{aligned}
& {\left[\varepsilon \xi^{(0)}+1\right]\left(\xi^{(0)^{\prime \prime}}+\varepsilon \xi^{(1)^{\prime \prime}}\right)} \\
& +\frac{1}{2} \varepsilon \xi^{(0)^{\prime 2}}+\xi^{(0)}+\varepsilon \xi^{(1)}=0,
\end{aligned}
$$

where second and higher order terms in $\varepsilon$ have been neglected. By carrying out further algebra and by still retaining only first-order terms in $\varepsilon$, we write:

$$
\begin{aligned}
& \xi^{(0)^{\prime \prime}}+\xi^{(0)}+\varepsilon\left(\xi^{(1)^{\prime \prime}}+\xi^{(1)}\right. \\
& \left.+\xi^{(0)} \xi^{(0)^{\prime \prime}}+\frac{1}{2} \xi^{(0)^{\prime 2}}\right)=0
\end{aligned}
$$

Therefore, the above equation splits into two different parts, one for the unperturbed solution $\xi_{0}$ only, one for the perturbed term $\xi_{1}$, so that, we have:

$$
\left.\xi^{(0)}\right)^{\prime \prime}+\xi^{(0)}=0
$$



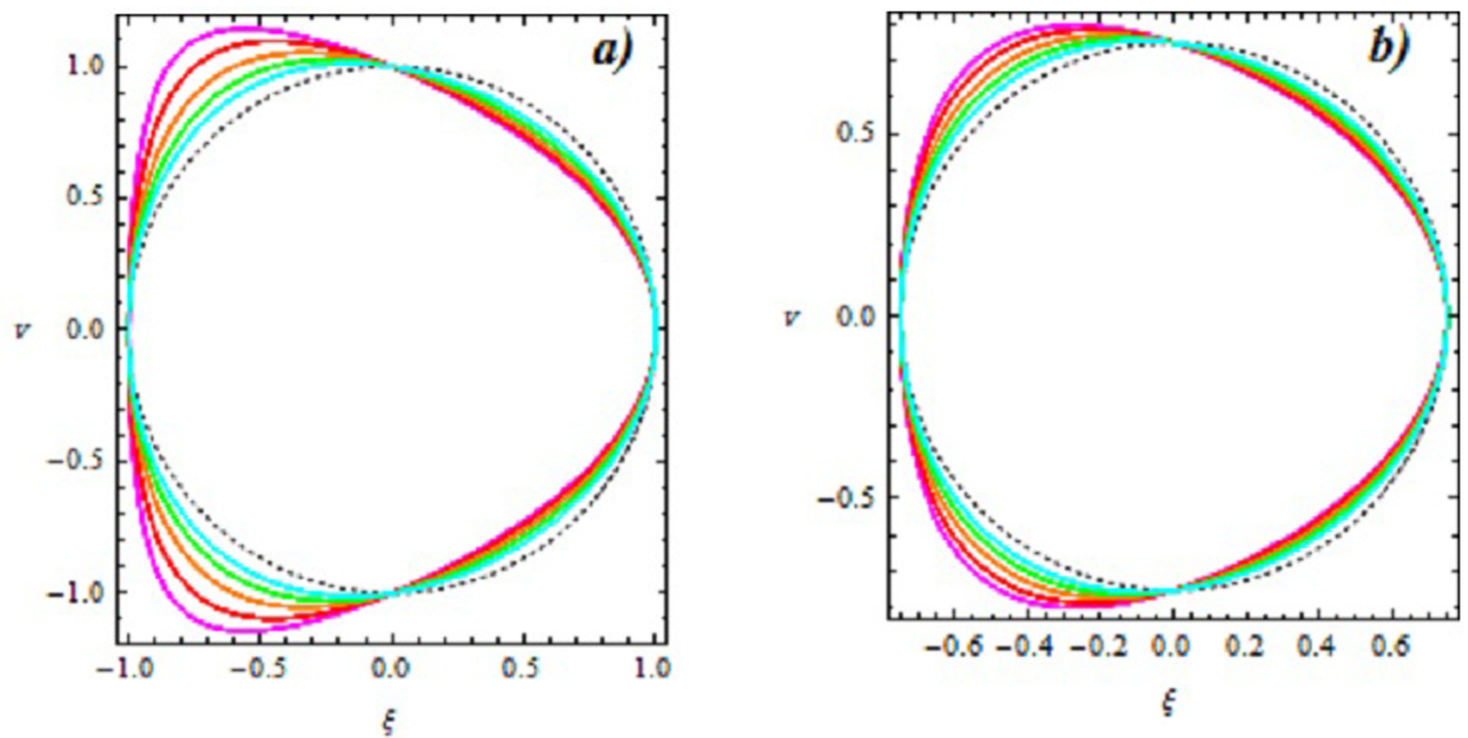

Figura 2: Phase-plane representation of the solution of the problem of communicating vessels. The initial conditions are the following: a) $\xi_{0}=1.0, v=0$; b) $\xi_{0}=0.75, v=0$. The colours magenta, red, orange, green cyan correspond to the following values of $\gamma: 0.15,0.25,0.40,0.55,0.70$. The dashed black circle corresponds to the solution for $\gamma=1$. Periodicity of the solution can be argued by the closing of all curves.

$$
\xi^{(1)^{\prime \prime}}+\xi^{(1)}=-\left(\xi^{(0)} \xi^{(0)^{\prime \prime}}+\frac{1}{2} \xi^{(0)^{\prime 2}}\right) .
$$

Notice that the differential equation (23a) describes free harmonic oscillations, whose solution is

$$
\xi^{(0)}(\tau)=A \sin (\tau+\phi),
$$

where the constants $A$ and $\phi$ are to be determined by means of the initial conditions $(19)$ applied to the solution $\xi(\tau)$. However, notion of the solution to equation (23a) is necessary to solve equation (23b). In this way, by substituting equation (24) into equation (23b) we obtain a differential equation describing the motion of a forced harmonic oscillator:

$$
\xi^{(1)^{\prime \prime}}+\xi^{(1)}=\frac{A^{2}}{4}[1-3 \cos (2 \tau+2 \phi)] .
$$

where a higher harmonic term appears. A general solution to equation (25) can be found by writing it as the sum of the homogeneous and particular solutions as follows:

$$
\xi^{(1)}(\tau)=B \sin (\tau+\psi)+\frac{A^{2}}{4}[1+\cos (2 \tau+2 \phi)] .
$$

In this way, the approximate solution $\xi(\tau)$ is, according to equation (20), the following:

$$
\begin{aligned}
\xi(\tau) & =A \sin (\tau+\phi)+\varepsilon B \sin (\tau+\psi) \\
& +\varepsilon \frac{A^{2}}{4}[1+\cos (2 \tau+2 \phi)] .
\end{aligned}
$$

where $A, B, \phi$, and $\psi$ need to be evaluated according to $(19)$, so that:

$$
\begin{gathered}
\xi_{0}=A \sin \phi+\varepsilon B \sin \psi+\varepsilon \frac{A^{2}}{4}[1+\cos 2 \phi] ; \\
0=A \cos \phi+\varepsilon B \cos \psi+\varepsilon \frac{A^{2}}{2} \sin 2 \phi .
\end{gathered}
$$

We thus find the following solution by separating the zero-order and the first-order terms:

$$
A=\xi_{0} ; \quad B=0 ; \quad \phi=\frac{\pi}{2} ; \psi=0 .
$$

Finally, we write equation (27) as follows:

$$
\xi(\tau)=\xi_{0} \cos \tau+\varepsilon \frac{\xi_{0}^{2}}{4}[1-\cos 2 \tau] .
$$

By taking the derivative with respect to $\tau$, we can also write:

$$
v(\tau)=-\xi_{0} \sin \tau+\varepsilon \frac{\xi_{0}^{2}}{2} \sin 2 \tau .
$$

We can thus rewrite these solutions in terms of the original variable $y_{1}$ :

$$
\begin{aligned}
y_{1}(t) & =\lambda\left\{1+\xi_{0} \cos \left(\sqrt{\frac{g}{\lambda}} t\right)\right. \\
& \left.+\varepsilon \frac{\xi_{0}^{2}}{4}\left[1-\cos \left(2 \sqrt{\frac{g}{\lambda}} t\right)\right]\right\} .
\end{aligned}
$$


By taking the derivative with respect to $t$, we can also write:

$$
\dot{y}_{1}(t)=-\lambda \xi_{0} \sqrt{\frac{g}{\lambda}} \sin \left(\sqrt{\frac{g}{\lambda}} t\right)\left[1-\varepsilon \xi_{0} \cos \left(\sqrt{\frac{g}{\lambda}} t\right)\right] .
$$

The constant $\xi_{0}$ is defined in equation $\sqrt{19}$ and the quantities $y_{2}$ and $\dot{y}_{2}$ can be known by means of equation (9).

The above solutions are periodic. In fact, we can now prove that the maximum deviation from a circular path in the phase plane of the functions $\xi$ and $v$ is a first-order term in $\varepsilon$. By considering equation (30) and (31) we can see that

$$
\xi^{2}+v^{2}=\xi_{0}^{2}-\varepsilon \xi_{0}^{3} \cos \tau \sin ^{2} \tau .
$$

Let us now consider the radial distance $\Delta r$ from the circumference $\xi^{2}+v^{2}=\xi_{0}^{2}$ in the phase plane and the curve in (34):

$$
\Delta r=\xi_{0}\left|1-\sqrt{1-\varepsilon \xi_{0} \cos \tau \sin ^{2} \tau}\right| .
$$

To first order in $\varepsilon$, we can write:

$$
\Delta r \approx \varepsilon \frac{\xi_{0}^{2}}{2}|\cos \tau| \sin ^{2} \tau .
$$

The maximum value of $\Delta r$ can be found by maximizing the time dependent term in (36), obtaining:

$$
\Delta r=\varepsilon \frac{\xi_{0}^{2}}{3 \sqrt{3}}
$$

Therefore, the effects due to non-linearity of the ordinary differential equation (17) are rather weak when very small values of $\varepsilon$ are considered. A comparison between the numerical solutions of equation (18a) and (18b) and the analytical solutions (30) and (31) obtained by means of the above perturbation approach for $\varepsilon=0.1$ and $\varepsilon=0.2$ is shown in Fig $3 \mathrm{a}$ and $3 \mathrm{~b}$. The latter figures, as for Fig. 2a and $2 \mathrm{~b}$, have been obtained by means of the software Mathematica.

\section{Conclusions}

The celebrated system of communicating vessels, which also finds applications in other subjects than physics, has been seen to possess interesting dynamical properties. In the present work we have considered two cylindrical vessels containing a nonviscous fluid. The two cylinders are connected by
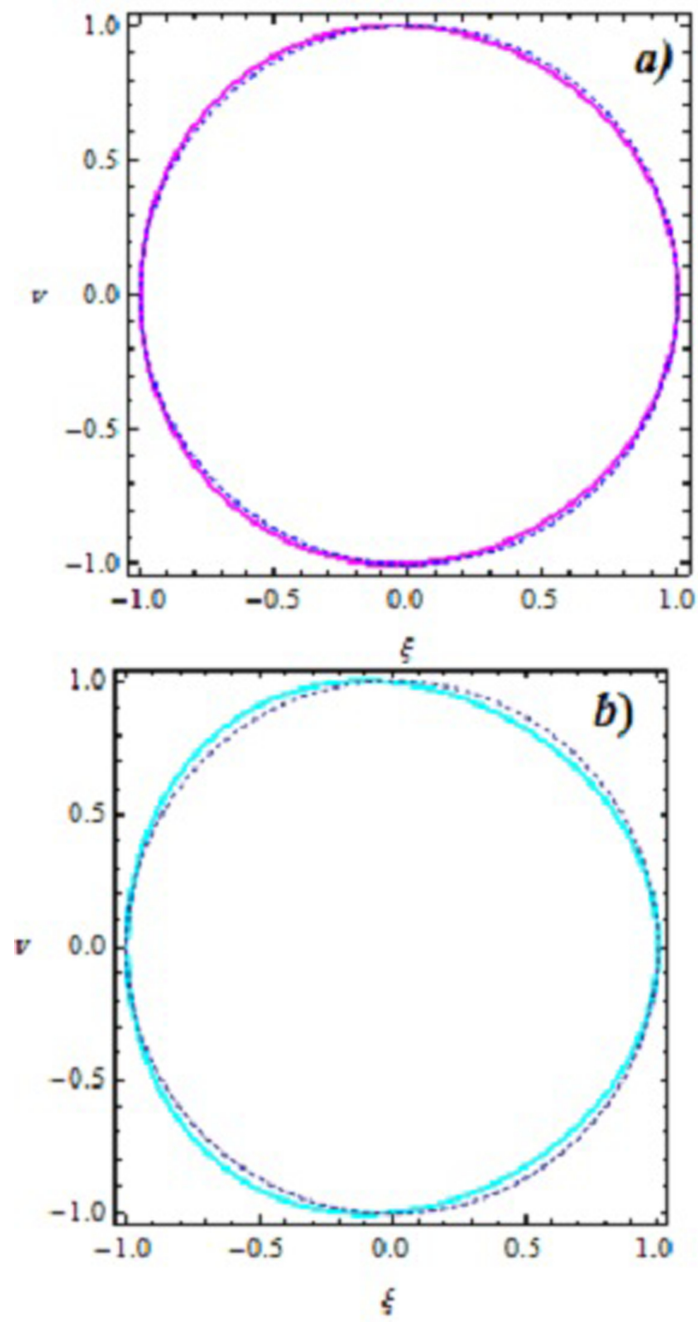

Figura 3: Phase-plane representation of the numerical (dashed) and approximated (full line) solution. The latter solution has been obtained by means of a perturbation analysis to first order in the parameter $\varepsilon$. The following values of $\varepsilon=1-\gamma$ have been chosen: $\varepsilon=0.10(\mathrm{a})$ and $\varepsilon=0.20(\mathrm{~b})$.

a small horizontal channel placed at their base. In the symmetric case, in which the two containers are perfectly identical, the system behaves like a harmonic oscillator, whose angular frequency $\omega_{0}$ is given by the following simple relation:

$$
\omega_{0}=\sqrt{\frac{g}{\lambda}}
$$

where $\lambda$ is the height, measured with respect to the bottom of the vessels, up to which the free surface of the liquid rises at equilibrium. Surprisingly, when we consider the asymmetric case, harmonic oscillations recede because of non-linear effects. The resulting non-linear differential equation preserves periodicity, 
as both a phase-plane analysis and a perturbation approach suggest.

As for the didactical value of the present work, we may affirm that the present study could be useful to students approaching the topic of nonlinear dynamical systems for the first time. In fact, the non-linear effects in the dynamical properties of the system can be seen either by means of a phase-plane analysis or by a perturbation approach. Both methods confirm periodic behavior of the system in the presence of non-linearity. Moreover, as a starting point students can rely on the harmonic oscillator mechanical equivalent system when the parameter $\varepsilon=1-S_{1} / S_{2}$, defining the degree of asymmetry between the sections $S_{1}$ and $S_{2}$ of the two cylindrical vessels, is zero. Nonlinear effects can be seen to gradually arise as $\varepsilon$ increases. Finally, students may notice that, while analytic solution of the problem is possible by means of a perturbation approach for small values of the parameter $\varepsilon$, numerical analysis is necessary for value of $\varepsilon$ approaching one.

\section{Referências}

[1] D. Halliday, R. Resnick and J. Walker, Fundamentals of Physics (Wiley and Sons, New York, 2005), 7th ed.

[2] F. van Waarden, in: Advancing Socio-Economics: An Institutionalist Perspective, edited by J.R. Hollingsworth, K.H. Müller and E.J. Hollingsworth (Rowman and Littlefield Publishers, Lanham, 2005), chapter 9.

[3] P.A. Tipler, Physics (Worth Publishers Inc., New York, 1980), chapter 14.

[4] R.J. Donnelly and O. Penrose, Phys. Rev. 103, 1137 (1956).

[5] M.J. Smith, J.J. Kobine and F.A. Davidson, Proc. R. Soc. A 464, 905 (2008).

[6] S.L.T. de Souza, I.L. Caldas, R.L. Viana, J.M. Balthazar and R.M.L.R.F. Brasil, Journal of Sound and Vibration 289, 987 (2006).

[7] R. De Luca and P. Desideri, Eur. J. Phys. 34, 189 (2013). 